\title{
A UNIT INTERPRETATION OF MATRIX CONVERGENCE ${ }^{1}$
}

\author{
William C. Wedley* and Eng U. Choo \\ Simon Fraser University \\ 8888 University Drive \\ Burnaby, B. C., CANADA, V5A 1S6 \\ Email: wedley@sfu.ca, choo@sfu.ca
}

\begin{abstract}
Raising the paired comparison matrix to powers until column ratios stabilize is an efficient and common method for calculating the principle eigenvector. The disadvantage of this method is that higher order calculations are difficult to comprehend. This paper suggests a modification of the process that averages the higher order calculations in a manner that maintains the matrix in its original units. It also compares additive aggregation to geometric aggregation and hierarchic composition.
\end{abstract}

Keywords: Matrix multiplication, eigenvector, convergence, aggregation

\section{Introduction}

The basic premise of AHP/ANP is that a ratio scale can be derived from the paired comparisons of $n$ objects that possess a ratio property. Each comparison $a_{i / j}$ is between the magnitudes of two objects ( $i, j$ ) with the smaller magnitude (j) as the unit of comparison. A total of $n(n-1) / 2$ such comparisons are performed, with the reciprocal value $1 / \mathrm{a}_{\mathrm{i} / \mathrm{j}}=\mathrm{a}_{\mathrm{j} / \mathrm{i}}$ representing a fundamental value when the object with the larger magnitude is used as the unit of measure. Along $w$ ith diagonal values $a_{i / i}=1$, these estimates are placed in an nxn matrix in which each column has the same unit of measure. Although each comparison value is an estimated ratio scale for two objects, the overreaching objective is to derive a scale that includes all $\mathrm{n}$ objects. A common procedure in AHP/ANP is to perform continued matrix multiplication until the ratios between objects of any column stabilize. The stabilized ratios coincide with the right eigenvector that is used as the derived measurement scale.

As will be shown, the row x column process of matrix multiplication is a type of arithmetic aggregation of indirect estimates of cell values. That aggregation can be re-expressed as an average that maintains the unit of the original matrix, thereby avoiding difficult interpretations of higher order calculations. The next sections outline the nature of the comparison matrix, matrix multiplication, and traits of the convergence process. The paper concludes by discussing anomalies with other procedures used in AHP.

\section{The Nature of a Comparison Matrix}

Figure 1 illustrates the layout and example of a comparison matrix. In order to emphasize that the cell entries are ratio estimates of the value of object $i$ given object $j$ as the unit of measure, we have used $a_{i j}$ notation rather than the usual $\mathrm{a}_{\mathrm{ij}}$ matrix specification. Notice from the layout of the matrix that all values in each column have a common unit. Therefore, each column in itse lf is a ratio scale of the $\mathrm{n}$ objects based upon a different unit of measure. If the DM is perfectly consistent when making the comparisons, then we could take any column as our measurement scale that spans the n objects. Perfect consistency

\footnotetext{
${ }^{1}$ The authors thank the Natural Sciences and Engineering Research Council of Canada for financial support related to this project (RGPIN123621 and RGPIN8432). * Corresponding author
} 
occurs when $\mathrm{a}_{\mathrm{i} / \mathrm{j}} \mathrm{a}_{\mathrm{j} / \mathrm{k}}=\mathrm{a}_{\mathrm{i} / \mathrm{k}}$ for all $\mathrm{i}, \mathrm{j}, \mathrm{k}=1 \ldots \mathrm{n}$. In the example shown, the matrix is not consistent. Some inconsistency is usual since the DM is seldom able to make all comparisons with perfect accuracy.

\begin{tabular}{|l|l|l|l|}
\hline $\mathrm{a}_{1 / 1}$ & $\mathrm{a}_{1 / 2}$ & $\mathrm{a}_{1 / 3}$ & $\mathrm{a}_{1 / 4}$ \\
\hline $\mathrm{a}_{2 / 1}$ & $\mathrm{a}_{2 / 2}$ & $\mathrm{a}_{2 / 3}$ & $\mathrm{a}_{2 / 4}$ \\
\hline $\mathrm{a}_{3 / 1}$ & $\mathrm{a}_{3 / 2}$ & $\mathrm{a}_{3 / 3}$ & $\mathrm{a}_{3 / 4}$ \\
\hline $\mathrm{a}_{4 / 1}$ & $\mathrm{a}_{4 / 2}$ & $\mathrm{a}_{4 / 3}$ & $\mathrm{a}_{4 / 4}$ \\
\hline
\end{tabular}

\begin{tabular}{c|c|c|c|c|}
\multicolumn{1}{c}{ A } & \multicolumn{1}{c}{ B } & \multicolumn{1}{c}{ C } & \multicolumn{1}{c}{$\mathrm{D}$} \\
\cline { 2 - 5 } $\mathrm{A}$ & 1 & 2 & 2.8 & 3 \\
\hline $\mathrm{B}$ & 0.5 & 1 & 1.2 & 1.8 \\
\hline $\mathrm{C}$ & 0.357 & 0.833 & 1 & 1.4 \\
\hline $\mathrm{D}$ & 0.333 & 0.556 & 0.714 & 1 \\
\hline
\end{tabular}

Figure 1 Layout and example of a reciprocal pairwise comparison matrix

There are three general ways to interpret comparison estimates. The first and more general interpretation is that they estimate the fixed absolute value between two objects. Even if both objects are measured in terms of some other unit, the ratio between them should always be the same. The second interpretation is that each comparison itself is a little scale that measures the property under consideration. For example, $\mathrm{a}_{1 / 4}$ is a scale where $\mathrm{D}=1$ (the unit) and $\mathrm{A}=3$ while the reciprocal, $\mathrm{a}_{4 / 1}$, has $\mathrm{A}=1$ (the unit) and $\mathrm{D}=1 / 3$. The third and more subtle interpretation is that each comparison, like exchange rates between currencies, is the conversion factor between one unit of measure and another. For example, if we have object $\mathrm{C}$ measured in terms of $A\left(a_{3 / 1}\right)$ and we wish to measure $C$ in terms of object $D\left(a_{3 / 4}\right)$, then the conversion factor between $A$ and $D\left(a_{1 / 4}\right)$ could be used to achieve the transformation $\left(a_{3 / 1} * a_{1 / 4}=a_{3 / 4}\right)$.

Because the DM is not capable of being perfectly accurate, it generally occurs that $\mathrm{a}_{\mathrm{i} / \mathrm{j}} \mathrm{a}_{\mathrm{j} / \mathrm{k}} \sim \mathrm{a}_{\mathrm{i} / \mathrm{k}}$. In other words, the DM's direct estimate $\left(a_{\mathrm{i} / \mathrm{k}}\right)$ differs from an indirect estimate $\left(\mathrm{a}_{\mathrm{i} / \mathrm{j}}{ }^{*} \mathrm{a}_{\mathrm{j} / \mathrm{k}}\right)$ calculated by converting from the unit of object $\mathrm{j}$ to the unit of object $\mathrm{k}$. In matrix multiplication, several different routes are available for calculating these indirect estimates.

\section{Achieving Ratio Stability via Matrix Multiplication.}

Let $\mathrm{T}$ be an estimated pairwise comparison matrix. Using matrix multiplication, ratio priorities are derived from the comparison matrix $\mathrm{T}$ by the following limit:

$$
\operatorname{Lim}_{k \rightarrow \infty} \frac{T^{k} e}{e^{T} T^{k} e}
$$

where $\mathrm{e}^{\mathrm{T}}=[1, \ldots, 1]$ is the unity $\mathrm{n}$-vector (Saaty, 2003). The unity vectors determine the row sum in the numerator and the sum of those row sums in the denominator. This process expresses priorities as a unitsum vector.

In addition to the above process, Saaty (2008) points out that the eigenvector solution is independent of whether all the elements of $\mathrm{T}$ are multiplied by the same constant or not. Thus, (1) can be re-written as:

$$
\operatorname{Lim}_{k \rightarrow \infty} \frac{b T^{k} e}{e^{T} b T^{k} e}
$$

where $\mathrm{b}$ is a positive scalar that proportionally transforms all cell values to a different unit of measure. Alternatively, the same effect is achieved if (1) is re-written

$$
\operatorname{Lim}_{k \rightarrow \infty} \frac{T^{k} B e}{e^{T} T^{k} B e}
$$


where $\mathrm{B}$ is a diagonal matrix with an arbitrary constant $(\mathrm{b}>0)$ on the diagonal that proportionally transforms all column values to a different unit of measure.

To comprehend what is happening when $\mathrm{T}$ is raised to higher powers, it is worthwhile to look at the notation and the results for calculating $\mathrm{T}^{2}$. First, consider just the comparison value for $\mathrm{a}_{1 / 3}$ of $\mathrm{T}$ that has a direct comparison value of 2.8. Recall that 2.8 and other values in the third column are expressed in the unit of object $\mathrm{C}$. Thus, the magnitude of $\mathrm{A}$ is estimated to be 2.8 times larger than the magnitude of $\mathrm{C}$. Getting cell $\mathrm{a}_{1 / 3}$ of $\mathrm{T}^{2}$ involves multiplying row 1 times column 3 and summing the results.

$$
\begin{aligned}
\mathrm{a}_{1 / 1} * \mathrm{a}_{1 / 3}+\mathrm{a}_{1 / 2} * \mathrm{a}_{2 / 3}+\mathrm{a}_{1 / 3} * \mathrm{a}_{3 / 3}+\mathrm{a}_{1 / 4} * \mathrm{a}_{4 / 3} \quad & =1 * 2.8+2 * 1.2+2.8 * 1+3 * 0.714 \\
& =2.8+2.4+2.8+2.14=10.14
\end{aligned}
$$

Notice that the synthesis is a summation of two direct estimates $\left(a_{1 / 1} * a_{1 / 3}, a_{1 / 3} * a_{3 / 3}\right)$ and two indirect estimates $\left(a_{1 / 2} * a_{2 / 3}, a_{1 / 4} * a_{4 / 3}\right)$. Each component of the summation is a convers ion from the $n$ different units of each row $\left(a_{1 / 1}, a_{1 / 2}, a_{1 / 3}, a_{1 / 4}\right)$ to the unit of the target cell $\left(a_{1 / 3}\right)$. Accordingly, matrix multiplication to get $T^{2}$ is the component-wise summation of $n$ estimates of $a_{i j}$ from $n$ source units of measure.

The next matrix multiplication to get $\mathrm{a}_{1 / 3}$ of $\mathrm{T}^{3}$ involves 4 estimates from 4 source units that already contain 4 estimates themselves. Thus $\mathrm{a}_{1 / 3}$ in $\mathrm{T}^{2} * \mathrm{~T}=\mathrm{T}^{3}$ becomes $\mathrm{a}_{1 / 3}=\left(\mathbf{a}_{1 / 1} * \mathrm{a}_{1 / 3}+\mathbf{a}_{1 / 2} * \mathrm{a}_{2 / 3}+\mathbf{a}_{1 / 3} * \mathrm{a}_{3 / 3}+\right.$ $\mathbf{a}_{1 / 4} * \mathrm{a}_{4 / 3}$ ) where the bold faced items are already composed of 4 estimates. In total, $\mathrm{T}^{3}$ has $\mathrm{n}^{\mathrm{k}-1}=4^{2}$ estimates for each cell. At higher powers the number of cell estimates increase exponentially -- 4 for $\mathrm{T}^{2}, 16$ for $\mathrm{T}^{3}$, 64 for $\mathrm{T}^{4}$, etc.

Figure 2 presents the results of matrix multiplication at $T^{2}$ and $T^{3}$. Notice that cell $a_{1 / 3}$ in $T^{2}$ has the calculated value 10.14. This value is certainly not an estimate of the comparison value of objects $\mathrm{A} / \mathrm{C}$, because the A/C estimates shown in (4) are closer to the direct estimate, 2.8. From (4), however, we can see that the $a_{1 / 3}$ value in $T^{2}$ is a summation or concatenation of 4 estimates of $A / C$. Accordingly, the row items in $\mathrm{T}^{2}$ should be interpreted as an abstract object that is 4 times as large as the original row item (i.e. $\mathrm{a}_{1 / 3}$ in $\mathrm{T}^{2}$ is $4 \mathrm{~A} / 1 \mathrm{C}$ ). This fact is illustrated in Figure 2 by showing the row object as 4 times the magnitude of the previous matrix object. Thus, by $\mathrm{T}^{3}$, the unit of measure in each columnar scale is still one unit of the column object, but the abstract concatenated object in each row is 16 times larger than the unit, 64 times larger at $\mathrm{T}^{4}$, etc. The number of summed estimates for each step is $\mathrm{n}^{\mathrm{k}-1}$.

$T$

\begin{tabular}{c|c|c|c|c|}
\multicolumn{1}{c}{$\mathrm{A}$} & \multicolumn{1}{c}{$\mathrm{B}$} & \multicolumn{1}{c}{$\mathrm{C}$} & \multicolumn{1}{c}{$\mathrm{D}$} \\
\hline $\mathrm{A}$ & $\mathrm{A}$ & 2 & 2.8 & 3 \\
\hline $\mathrm{B}$ & 0.50 & 1 & 1.2 & 1.8 \\
\hline $\mathrm{C} 0.357$ & 0.833 & 1 & 1.4 \\
\hline $\mathrm{D}$ & 0.333 & 0.556 & 0.714 & 1 \\
\hline
\end{tabular}

$\mathrm{T}^{2}$

\begin{tabular}{c|c|c|c|c|}
\multicolumn{1}{c}{$\mathrm{A}$} & \multicolumn{1}{c}{$\mathrm{B}$} & \multicolumn{1}{c}{$\mathrm{C}$} & $\mathrm{D}$ \\
\cline { 2 - 5 } $4 \mathrm{~A}$ & 4.000 & 8.000 & 10.143 & 13.520 \\
\hline $4 \mathrm{C}$ & 2.029 & 4.000 & 5.086 & 6.780 \\
\cline { 2 - 5 } $4 \mathrm{C}$ & 1.598 & 3.159 & 4.000 & 5.371 \\
\hline $4 \mathrm{D}$ & 1.200 & 2.373 & 3.029 & 4.000 \\
\hline
\end{tabular}

$T^{3}$

\begin{tabular}{c|c|c|c|c|}
\multicolumn{1}{c}{$\mathrm{c} A$} & \multicolumn{1}{c}{$\mathrm{B}$} & \multicolumn{1}{c}{$C$} & \multicolumn{1}{c}{$\mathrm{D}$} \\
\cline { 2 - 5 } $16 \mathrm{~A}$ & 16.129 & 31.963 & 40.600 & 54.120 \\
\cline { 2 - 5 } $16 \mathrm{~B}$ & 8.105 & 16.062 & 20.409 & 27.186 \\
\cline { 2 - 5 } $16 \mathrm{C}$ & 6.396 & 12.671 & 16.101 & 21.450 \\
\cline { 2 - 5 } & 4.801 & 9.518 & 12.092 & 16.110 \\
\hline
\end{tabular}

Figure 2 Comparison matrix values at $\mathrm{T}, \mathrm{T}^{2}$ and $\mathrm{T}^{3}$.

If we use (2) or (3) with $\mathrm{b}=1 /\left(\mathrm{n}^{\mathrm{k}-1}\right)$, then we re-scale the columns of $\mathrm{T}^{\mathrm{k}}$ back to $\mathrm{T}^{1}$ conditions with average values in the cells. This is illustrated in Figure 3. Notice in Figure 3 that each subsequent multiplication deflects cell values towards stable averages. Even by the third iteration, the ratio of object $\mathrm{A}$ to $\mathrm{B}$ in all columns of $1 / 16 \mathrm{~T}^{3}$ is 1.99 . As can be seen from Figure 3, the advantage of using $\mathrm{T}^{\mathrm{k}} / \mathrm{n}^{\mathrm{k}-1}$ is that successive matrix multiplications can be related back to the original units and magnitudes of $\mathrm{T}$. In this format, cell values can be observed between iterations to monitor the progression to stability. 


\begin{tabular}{l|c|c|c|c|}
\multicolumn{5}{c}{} \\
\multicolumn{1}{c}{$\mathrm{A}$} & \multicolumn{1}{c}{$\mathrm{B}$} & \multicolumn{1}{c}{$\mathrm{C}$} & \multicolumn{1}{c}{$\mathrm{D}$} \\
\hline $\mathrm{A}$ & $\mathrm{A}$ & 2 & 2.8 & 3 \\
\hline $\mathrm{B}$ & 0.500 & 1 & 1.2 & 1.8 \\
\hline $\mathrm{C}$ & 0.357 & 0.833 & 1 & 1.4 \\
\hline $\mathrm{D}$ & 0.333 & 0.556 & 0.714 & 1 \\
\hline
\end{tabular}
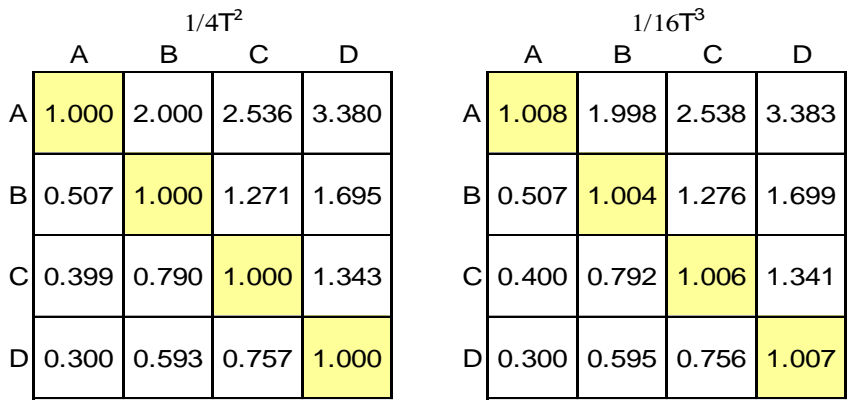

Figure 3 Comparison matrix values at $\mathrm{T}, 1 / 4 \mathrm{~T}^{2}$ and $1 / 16 \mathrm{~T}^{3}$

Using (1), (2) or (3), stability eventually evolves for the ratio of objects in any of the columns. Invariance of the ratio of any two objects is a property of ratio scales (Michell, 1997). When such invariance eventually occurs, we can conclude that sufficient conversion has taken place to average out any inaccuracy in the comparisons. The final stabilized weights normalized to unity are: $0.4552,0.2287$, 0.1805 , and 0.1355 for $\mathrm{A}, \mathrm{B}, \mathrm{C}$ and $\mathrm{D}$ respectively. We can look upon these eigenvector values as the average of many estimates of column values.

\section{Some Interesting Traits of Matrix Converge nce.}

In passing, we should recognize some traits of the additive process used in matrix multiplication. The first is that additive synthesis destroys the reciprocal property that was inherent in the original comparison matrix. Notice that all $\mathrm{a}_{\mathrm{i} / \mathrm{i}}>1$ in $\mathrm{T}^{2}$ and $\mathrm{T}^{3}$. This occurs because we are no longer comparing one unit of each object. For example, in $\mathrm{T}^{2}, \mathrm{a}_{1 / 2}=4 \mathrm{~A} / 1 \mathrm{~B}$ and the $\mathrm{a}_{2 / 1}=4 \mathrm{~B} / 1 \mathrm{~A}$. Even if we multiplied all $\mathrm{T}$ by $1 / \mathrm{n}$ to express the values back in terms of $\mathrm{T}^{1}$, the reciprocal relationship would not be maintained. Such nonreciprocity is common (e.g. cross exchange rates for currencies or actual estimates for reciprocal values) and can be easily handled in matrix multiplication.

A second trait of the additive process of matrix multiplication is that any rescaling via B in (3) must have all diagonal values equivalent in order to produce the same eigenvector solution as (1). For example, we could set

$$
\mathrm{b}_{\mathrm{j}}=\frac{1}{\mathrm{Te}}=\frac{1}{\sum_{\mathrm{i}=1}^{\mathrm{n}} \mathrm{a}_{\mathrm{i} / \mathrm{j}}}
$$

in B to normalize each column to sum to 1 . This would make it columnar stochastic like an ANP supermatrix, but the eigenvector solution would be different. The stabilized solution when $\mathrm{T}$ is converted to a stochastic matrix is $0.4566,0.2280,0.1756$ and 0.1397 for $\mathrm{A}, \mathrm{B}, \mathrm{C}$ and $\mathrm{D}$ respectively. This different solution suggests that that additive synthesis does not have the same homogeneity property that is associated with geometric aggregation. (Saaty \& Sodenkamp, 2009).

\section{Discussion}

Raising the comparison matrix to powers to achieve convergence is essentially a process of componentwise addition of $\mathrm{n}^{\mathrm{k}-1}$ matrices with an estimate $\mathrm{a}_{\mathrm{i} / \mathrm{j}}$ in each cell. For $\mathrm{T}^{2}$, this process is illustrated in Figure 4. For $\mathrm{k}>2$, the process would just be adding more of these estimate matrices. 

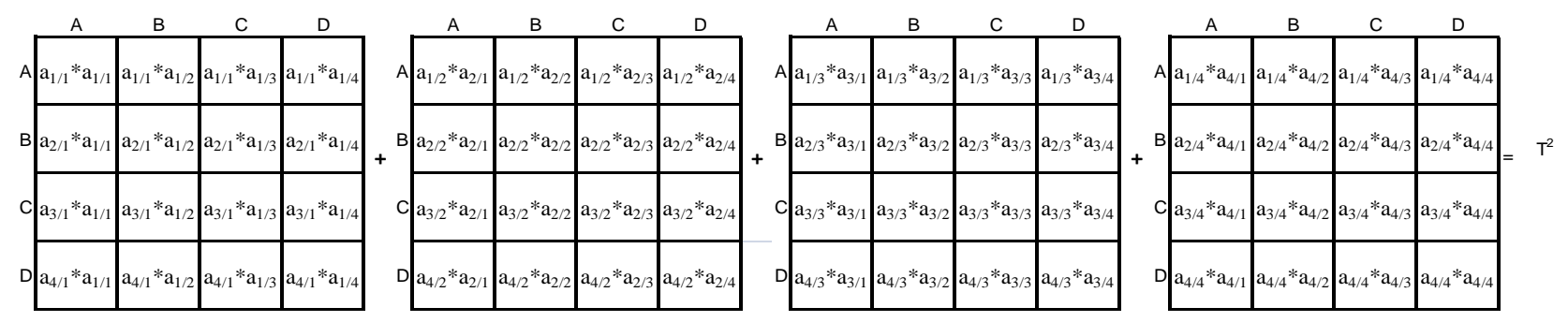

Figure 4 Component-wise addition of 4 estimates to get $\mathrm{T}^{2}$.

When viewed from this perspective of aggregating $\mathrm{n}^{\mathrm{k}-1}$ matrices, determining $\mathrm{T}^{\mathrm{k}}$ is the same as the problem of aggregating matrices of $\mathrm{k}$ individuals into a group matrix. For group decision making, however, the accepted AHP method for aggregating individual judgments is not an additive one. Instead, the geometric mean or weighted geometric mean is universally observed as the correct method (Aczel \& Saaty, 1983; Aczel \& Alsina, 1986; Forman \& Peniwati, 1998; Saaty \& Peniwati, 2008). The geometric mean has desirable properties such as reciprocal relationships, unanimity and homogeneity conditions (Saaty \& Sodenkamp, 2009).

It is obvious that there is an anomaly between group and eigenvector routines. Although both processes aggregate matrices, geometric aggregation is used for the group matrix, but additive aggregation is for the converged matrix. This is in spite of the fact that both processes aggregate cell estimates. It also leads to the irony that the group matrix from geometric aggregation is then subjected to additive aggregation to get the eigenvector.

Another anomaly is associated with the principle of hierarchic composition. Saaty (2003) points out that the principal eigenvector is the only priority vector that remains invariant under the principle of hierarchic composition. As displayed graphically in Figure 5, the hierarchical problem is to determine weights $\mathrm{w}_{\mathrm{i}}$, $\mathrm{i}=1,2 \ldots 4$ that correctly represent the relative dominance of the four objects (i.e. the unit for each cluster). As Saaty observes, only the eigenvector for relative weights produces the same relative weights for priorities $\left(\mathrm{p}_{\mathrm{i}}\right)$.

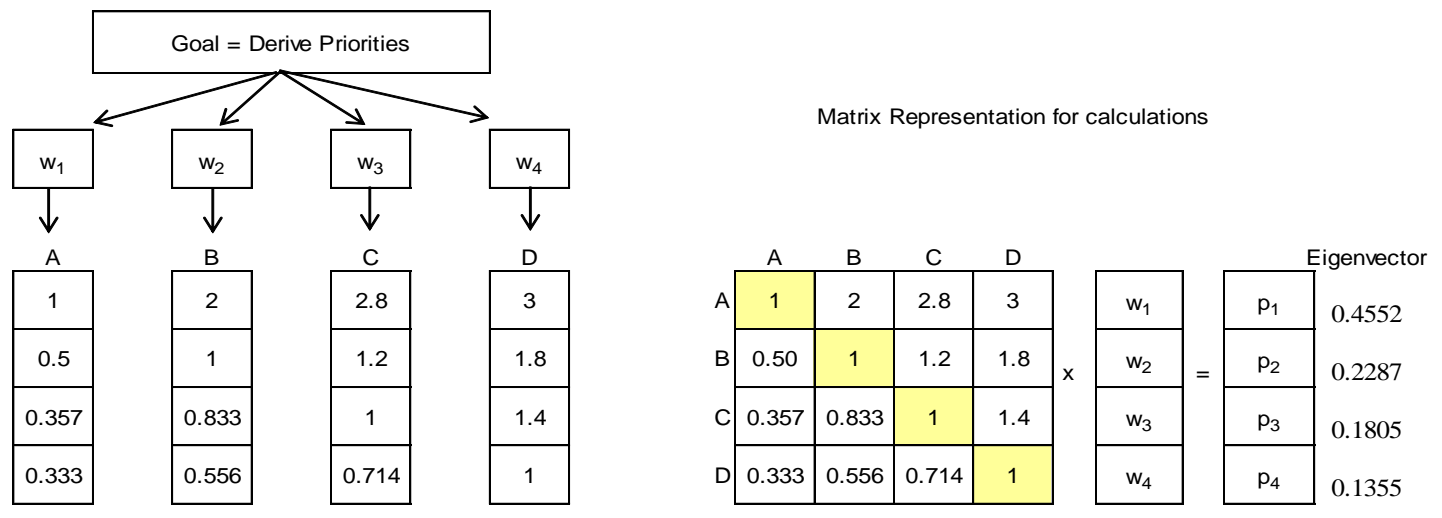

Figure 5 Hierarchic and Matrix representations for deriving prior ities

In hierarchic structuring, however, it is common practice to normalize local cluster values to unit-sum vectors. As pointed out above, the eigenvector solution to the stochastic matrix is different. This is not too surprising when we consider that the normalization to unity is equivalent to changing the unit of clusters to a common object (an abstract totality object that is the sum of the 4 objects). Since the unit of each column is the same (a totality), we should use equal weights (not eigenvector weights) when the normalized columns sum to one. This method of using a simple average with a totality unit is called 
Normalized Column Means (NCM). It has been reported to be effic ient and accurate (Bajwa et al, 2008, Zahedi, 1986). Why NCM should be an inferior method of hierarchical composition is not clear. Nor is it clear why geometric aggregation is inferior when it is used in group processes.

\section{Conclusion}

Matrix convergence of cell values can be interpreted as the simple average of many conversion estimates. At stability, the cell values in every column have the same ratio to each other. Invariance of ratios irrespective of the unit of measure is a trait of ratio scales. The eigenvector routine achieves this trait. The eigenvector routine, however, is not the only method that generates ratio stability. The geometric mean that is used in group aggregation has many desirable properties. And, the simple mean of unit sum columns (NCM) has proved to be quite accurate. Which aggregation method has the greatest efficacy is a topic that requires more investigation.

\section{REFERENCES}

Aczel, J. \& Alsina, C. (1986). On the synthesis of judgments. Socio-Economic Planning Sciences, 20, 333-339.

Aczel, J \& Saaty, T.L. (1983). Procedures for synthesizing ratio judgments. Journal of Mathematical Psychology, 27, 93-102.

Bajwa G., Choo E.U, \& Wedley W.C. (2008). Effectiveness analysis of deriving priority vectors from reciprocal pairw ise comparison matrices. Asia Pacific Journal of Operational Research 25: 279-299.

Forman, E. \& Peniwati, K. (1998). Aggregating individual judgments and priorities with the Analytic Hierarchy Process. European Journal of Operational Research, 108, 165-169.

Lin C.C. (2007). A revised framework for deriving priority values from pairwise comparison matrices. European Journal of Operational Research 176: 1145-1150.

Michell, J. (1997). Quantitative science and the definition of measurement in psychology. British Journal of Psychology, 88, 355-383.

Saaty T.L. (2003). Decision-making with the AHP: Why is the principal eigenvector necessary? European Journal of Operational Research, 145: 85-91.

Saaty, T.L. (2008). Relative measurement and its generalization in decision-making - Why pairwise comparisons are central in mathematics for the measurement of intangible factors: The Analytic Hierarchy/Network Process. RACSAM, Revista de la Real Academia de Ciencias Serie A: Matemáticas 102(2) 251-318.

Saaty, T.L. and Sodenkamp, M. (2009). Making decisions in hierarchic and network systems. International Journal of Applied Decision Sciences, 1, 1, 24-78.

Saaty, T.L., \& Peniwati, K. (2007). Group decision-making: Drawing out and reconciling differences. Pittsburgh, PA: RWS Publications.

Zahedi F. (1986). A simulation study of estimation methods in the Analytic Hierarchy Process. SocioEconomic Planning Sciences, 20: 347-354. 